\title{
microRNA-99a inhibits cell proliferation, colony formation ability, migration and invasion by targeting fibroblast growth factor receptor 3 in prostate cancer
}

\author{
DEYAO WU*, YUNFENG ZHOU*, HUIXING PAN, PING QU and JIAN ZHOU \\ Department of Urology, The Fourth Affiliated Hospital of Nantong Medical College, \\ Yancheng City First People's Hospital, Yancheng, Jiangsu 224001, P.R. China
}

Received April 9, 2014; Accepted August 6, 2014

DOI: $10.3892 / \mathrm{mmr} .2014 .2792$

\begin{abstract}
RNA-99a (miR-99a) was reported to be among the most frequently downregulated miRNAs in numerous types of human cancers, including prostate, bladder, hepatocellular and ovarian carcinoma, squamous cell carcinoma of the tongue, squamous cell lung carcinoma as well as childhood adrenocortical tumors. The aim of the present study was to determine the effects of miRNA-99a on cell proliferation, colony formation ability, migration and invasion in prostate cancer. Following transfection with miRNA-99a, cell viability, colony formation, cell migration and cell invasion assays were performed on prostate cancer cell lines, as well as western blot analysis and luciferase assays. miRNA-99a inhibited cell proliferation, colony formation ability, migration and invasion in DU145 and PC-3 cells, therefore indicating that miRNA-99a may have a tumor suppressive role in prostate cancer. In addition, the present study provided the first evidence that the mechanism of action of miRNA-99a may proceed by directly targeting fibroblast growth factor receptor 3 in prostate cancer. In conclusion, the results of the present study suggested that miRNA-99a may have potential use as a therapeutic target for the treatment of prostate cancer.
\end{abstract}

\section{Introduction}

Prostate cancer remains one of the most prevalent types of cancer in males (1). In the USA and Europe, prostate cancer is currently the second most common cause of cancer mortality

Correspondence to: Dr Jian Zhou, Department of Urology, The Fourth Affiliated Hospital of Nantong Medical College, Yancheng City First People's Hospital, 15 Yuehe Road, Yancheng, Jiangsu 224001, P.R. China

E-mail: luqiang0405@163.com

\section{${ }^{*}$ Contributed equally}

Key words: prostate cancer, fibroblast growth factor receptor 3, miR-99a, microRNA in males $>40$ years of age, and the third most common cause of cancer-associated mortality in males $(2,3)$. A total of 238,590 novel cases and 29,720 mortalities due to prostate cancer were estimated for 2013 in the USA (2). Although the etiology of this malignancy remains to be elucidated, several risk factors have been identified that are considered to contribute to prostate carcinogenesis, including hereditary and environmental components; however, age, race and family history are the only well-established risk factors (4). Early-stage cancers are managed using various treatments, including radical prostatectomy, radiation or hormone ablation therapy. Prostate cancer is only temporarily manageable using hormone deprivation, it will inevitably become resistant to hormonal therapy, following which there is currently no effective treatment (5). Therefore, understanding the molecular basis of prostate cancer and the development of novel treatments are crucial for improving the survival rates of prostate cancer patients.

MicroRNAs (miRNAs) are a type of endogenously expressed small, non-coding, single-stranded RNAs. miRNAs are able to negatively regulate gene expression through binding to the 3 ' untranslated region (3'UTR) of their target messenger RNAs (mRNAs) (6). In mammalian cells, miRNAs affect gene silencing via translational inhibition and mRNA degradation; an individual miRNA is capable of regulating numerous distinct mRNAs. miRNAs have been estimated to regulate $\sim 30 \%$ of the human genome (7). Hundreds of evolutionarily conserved miRNAs have been identified in plants, animals and viruses $(7,8)$. These molecules have been reported to control fundamental cell functions, including proliferation, apoptosis and differentiation; this therefore indicated that they may have a role in carcinogenesis (9-11). Numerous studies have illustrated that miRNAs are aberrantly expressed in human malignancies, such as prostate cancer (12-14). Upregulated miRNAs in cancer may function as oncogenes through the negative regulation of tumor suppressor genes (15). By contrast, downregulated miRNAs have been reported to function as tumor suppressor genes and inhibit cancer development through the regulation of oncogenes (16). Therefore, the identification of the target of miRNAs may be critical for understanding the function of miRNAs in cancer development and progression. It has also been suggested that miRNAs may be useful therapeutic targets for novel cancer therapies. 
miR-99a has been reported to be a frequently downregulated miRNA in numerous types of human cancers, including prostate, bladder, hepatocellular and ovarian carcinoma, squamous cell carcinoma of the tongue, squamous cell lung carcinoma as well as childhood adrenocortical tumors (17). A study by Sun et al (18) reported that miR-99a suppressed the expression of prostate-specific antigen and prostate cancer cell proliferation by targeting switch/sucrose nonfermentable-related matrix-associated actin-dependent regulator of chromatin subfamily A member 5 and subfamily D member 1 as well as the mechanistic target of rapamycin (mTOR). The aim of the present study was to determine the effects of miR-99a on cell proliferation, colony formation ability, migration and invasion in prostate cancer cell lines. In addition, the effect of miR-99a expression on fibroblast growth factor receptor 3 (FGFR3) was examined.

\section{Materials and methods}

Cells and culture conditions. The human prostate cancer cell lines DU145 and PC-3 were obtained from the Shanghai Institute of Biochemistry and Cell Biology (Shanghai, China). DU145 and PC-3 cells were cultured in RPMI 1640 medium (Gibco-BRL, Grand Island, NY, USA) supplemented with $10 \%$ heat-inactivated fetal bovine serum (FBS; Gibco-BRL) under a humidified atmosphere of $5 \% \mathrm{CO}_{2}$ at $37^{\circ} \mathrm{C}$. To propagate spheres in vitro, spheres were collected by gentle centrifugation (200 x g), dissociated into single cells, and then cultured to generate the next generation of spheres.

Transfection. Mature miR-99a mimics and miRNA mimics negative control (NC) were designed and synthesized by GenePharma(Shanghai,China).The sequence of miR-99amimics was 5'-AACCCGUAGAUCCGAUCUUGUG-3'. The sequence of NC mimics was 5'-UUCUCCGAACGUGUCACGUTT-3'. Cells were transfected using Lipofectamine 2000 (Invitrogen Life Technologies, Carlsbad, CA, USA) according to the manufacturer's instructions.

Quantitative polymerase chain reaction ( $q P C R$ ) for miR-99a. Total cellular RNA was extracted using TRIzol reagent (Invitrogen Life Technologies). The RNA was stored in diethylpyrocarbonate-treated water at $-80^{\circ} \mathrm{C}$ and the quantity and quality of the samples were evaluated prior to use with an ND-1000 NanoDrop spectrophotometer (NanoDrop Technologies, Wilmington, DE, USA). qPCR for miR-99a was performed using a SYBRgreen microRNA assay (Genepharm, Shanghai, China) according to the manufacturer's instructions. qPCR was performed using a 7300 Real-time PCR System (Applied Biosystems, Foster City, CA, USA) with an miR-99a primer set and double strand binding dye SYBRgreen. All primers were obtained from the TaqMan miRNA assays. GAPDH (Genepharm, Shanghai, China) was used as an internal control. Every sample was replicated three times. Data were analyzed by comparing $\mathrm{Ct}$ values.

Cell viability assay. Cell proliferation was determined using the MTT assay. The transfected cells (miR-99a mimics and NC) were seeded in a 96-well plate at a density of 3,000 cells per well. Cell viability assays were performed every $24 \mathrm{~h}$ for 5 days. In brief, $20 \mu \mathrm{l}$ MTT solution was added to each well and incubated at $37^{\circ} \mathrm{C}$ for $4 \mathrm{~h}$. Plates were then centrifuged ( $\left.200 \mathrm{x} \mathrm{g}, 10 \mathrm{~min}\right)$, and the purple colored precipitates of formazan were dissolved in $200 \mu \mathrm{l}$ dimethyl sulfoxide. Absorbance was measured at $490 \mathrm{~nm}$ in an ELISA reader (Model 550; Bio-Rad, Richmond, CA, USA). The suppression rate was calculated using the formula: Suppression rate $=(1-O D m i R-145 / O D m i R-N C) \times 100 \%$. Proliferation curves were drawn on the basis of mean absorbance at each time-point. All the experiments were performed in triplicate.

Colony formation assay. The colony-forming ability of DU145 and PC-3 cells transfected with miR-99a was assessed using a colony formation assay. In brief, the transfected cells (miR-99a mimics and NC) growing in log phase were trypsinized and seeded into six-well plates with a density of 2,000 cells per well. The cells were kept in an incubator at $37^{\circ} \mathrm{C}$ for seven days. On day eight, the colonies were washed with phosphate-buffered saline (PBS), fixed with formalin (10\%; Beyotime Institute of Biotechnology, Haimen, China) and stained with methyl violet. Finally, the methyl violet dye was washed off with PBS. The number of colonies was counted using a microscope (Olympus IX53; Olympus Corporation, Tokyo, Japan). Colony-inhibition rate $=[1-($ number of colonies in experimental groups/control group)]x100\%; and colony-forming efficiency $=(1$-colony-inhibition rate), were calculated.

Cell migration and invasion assay. Cell migration and invasion were assayed using Transwell chambers $(8 \mu \mathrm{m}$; Costar, Cambridge, MA, USA). For the migration assay, the transfected cells (miR-99a mimics and NC) growing in the log phase were trypsinized and resuspended as single cell solutions. A total of $1 \times 10^{5}$ cells per well were placed into the upper chamber cultured in medium with $2 \%$ FBS, while $500 \mu 1$ medium containing $20 \%$ FBS was added to the lower chamber. For the invasion assay, a Transwell chamber coated with Matrigel ${ }^{\circledR}(\mathrm{BD}$ Biosciences, San Jose, CA, USA) and a total of $1 \times 10^{5}$ cells were seeded into the upper chamber, while the bottom chamber was incubated with $500 \mu \mathrm{l}$ medium containing $20 \%$ serum. Cells were incubated for $12 \mathrm{~h}$ for the migration assay and $24 \mathrm{~h}$ for the invasion assay. At the end of the experiments, the cells on the upper surface of the membrane were removed, and the migrated cells were fixed with $100 \%$ methanol (Beyotime Institute of Biotechnology) for two min, stained in $0.5 \%$ crystal violet (Beyotime Institute of Biotechnology) for two min, rinsed in PBS and then subjected to microscopic inspection (magnification, x200). Five visual fields of each insert were randomly selected and counted under a light microscope (Olympus IX53). Each condition was assayed in triplicate and each experiment repeated at least three times.

Western blot analysis. Primary antibodies used in the present study, including FGFR3 (rabbit, polyclonal) and $\beta$-actin (rabbit, monoclonal), were purchased from Bioworld Technology (Louis Park, MN, USA). Transfected cells were washed with ice cold PBS and lysed with $1 \%$ radioimmunoprecipitation assay lysis buffer (Beyotime Institute of Biotechnology) $72 \mathrm{~h}$ following transfection. The protein concentration was determined using the bicinchoninic acid assay kit (Beyotime Institute of Biotechnology). Equal amounts of the proteins were separated 

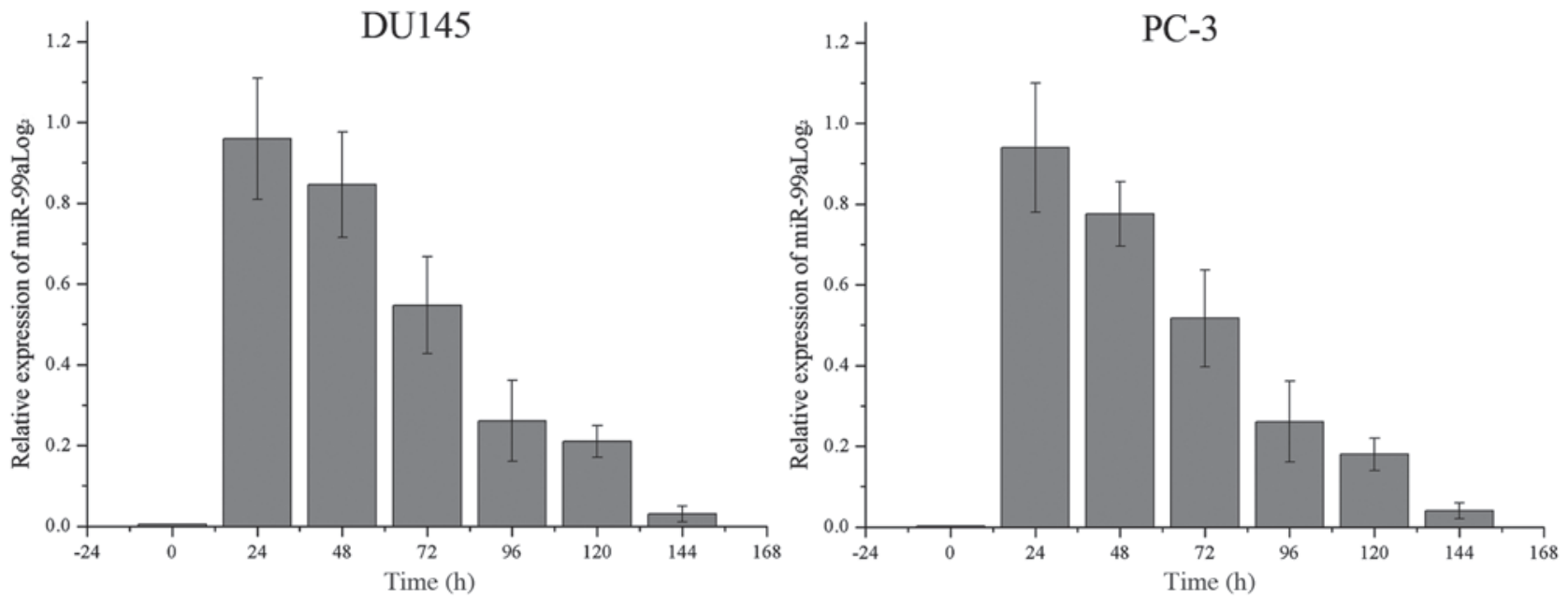

Figure 1. Increased levels of miR-99a following transfection into DU145 and PC-3 cells. miR-99a levels in DU145 and PC-3 cells peaked 24 h following transfection of miR-99a. Levels of miR-99a gradually declined but remained dramatically increased until $120 \mathrm{~h}$ following transfection compared to cells at 0 h. miR-99a, microRNA 99a. The error bars represent the standard deviation.

by $10 \%$ SDS PAGE (Beyotime Institute of Biotechnology) and transferred to polyvinylidene difluoride membranes (Beyotime Institute of Biotechnology). The membrane was blocked with $5 \%$ skimmed milk, followed by an overnight incubation at $4^{\circ} \mathrm{C}$ with a primary antibody at dilutions specified by the manufacturer's instructions. Following washing, the membrane was incubated with the corresponding horseradish peroxidase-conjugated secondary antibody (goat anti-rabbit; Bioworld Technology) in tris-buffered saline with Tween (Beyotime Institute of Biotechnology, China). The blot was then developed using an enhanced chemilluminescence solution (Pierce, Rockford, IL, USA) and images were captured using a FluorChem imaging system (Model number: 92-13779-00 FC2; Alpha Innotech, San Leandro, CA, USA).

Luciferase assay. The DU145 and PC-3 cells were transfected with $0.5 \mu \mathrm{g}$ reporter plasmid, $40 \mathrm{nmol} \mathrm{miR}-99 \mathrm{a}$ mimics or NC in a 12-well plate using Lipofectamine 2000 according to the manufacturer's instructions. The primers used for cloning FGFR3 mRNA 3'UTR were: Forward, GGGCTCGAGGGCCACTGGTCCCCAACAATGTG and reverse, GGGCGGCCGCCCAGTAACAGTACAGAACGA ACCAAC. Assays were performed using the Dual-Luciferase Reporter Assay system (Promega, Manheim, Germany) following $48 \mathrm{~h}$ of transfection. The firefly and renilla luciferase activities were measured using a TECAN Infinite 200 luminometer (Tecan, Männedorf, Switzerland). The firefly luciferase activity was normalized to the renilla luciferase activity for each transfected well. To determine whether miR-99a targets the FGFR3 3'-UTR, TARGETSCAN 5.2 (www.targetscan.org) and PICTAR (pictar.mdc-berlin.de) were used to assess the complementarity of miR-99a to the FGFR3 3'-UTR. Each reporter plasmid was transfected at least three times (on different days) and each sample was assayed in triplicate.

Statistical analysis. Values are presented as the mean \pm standard deviation. Statistical differences were analyzed using the Student's t test. Stata 10.0 software (StataCorp LP, College Station, TX, USA) was used for statistical analysis. $\mathrm{P}<0.05$ was considered to indicate a statistically significant difference between values.

\section{Results}

Relative miR-99a levels increase significantly following transfection into DU145 and PC-3 cells. The endogenous levels of miR-99a in DU145 and PC-3 cells as well as its levels following transfection of miR-99a were recorded every $24 \mathrm{~h}$. As shown in Fig. 1, miR-99a levels were significantly increased until $\sim 120 \mathrm{~h}$ following transfection in the two cell lines. The levels of miR-99a declined gradually following a peak at $24 \mathrm{~h}$.

miR-99a reduces cell proliferation and colony formation ability of DU145 and PC-3 cells. An MTT assay was used in order to investigate the influence of miR-99a on cell proliferation. Upregulation of miR-99a significantly inhibited cell proliferation (Fig. 2A). MTT assays revealed that following $144 \mathrm{~h}$ of treatment, the growth suppression rate of miR-99a reached $33.42 \pm 2.5 \%$ in DU145 cells and $29.13 \pm 2.8 \%$ in PC-3 cells.

A colony formation assay was used to determine the effect of miR-99a upregulation on colony formation ability. As shown in Fig. 2B, the relative colony-formation efficiency was significantly reduced to $59.8 \pm 5.6 \%$ in DU145 cells and $38.7 \pm 6.1 \%$ in PC-3 cells. These results therefore indicated that miR-99a may have an important role in the regulation of prostate cancer DU145 and PC-3 cell lines.

miR-99a suppresses cell migration and invasion in prostate cancer DU145 and PC-3 cells. A Transwell assay was performed in order to measure the effect of miR-99a on tumor cell migration and invasion. Migration of miRNA-99a-transfected cells was significantly decreased to $47.14 \pm 5.38 \%$ in DU145 cells and $51.95 \pm 6.18 \%$ in PC-3 cells (Fig. 3A). In the invasion assay (Fig. 3B), the miR-99a groups were found to have decreased cell invasion of $55.28 \pm 6.42 \%$ in DU145 cells and $65.76 \pm 7.37 \%$ in PC-3 cells. These results indicated that miR-99a reduced migration and invasion in prostate cancer DU145 and PC-3 cell lines. 


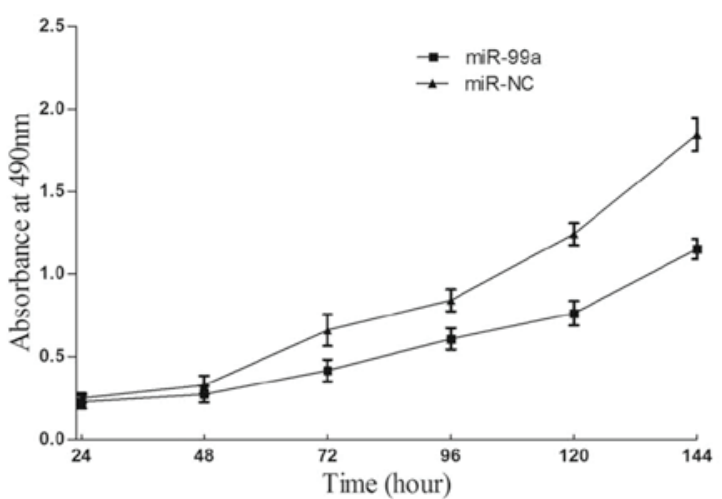

DU145

\section{B}

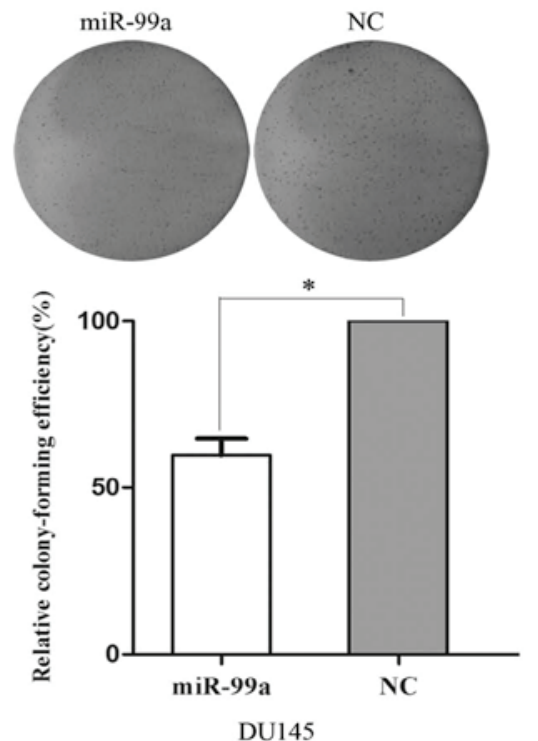

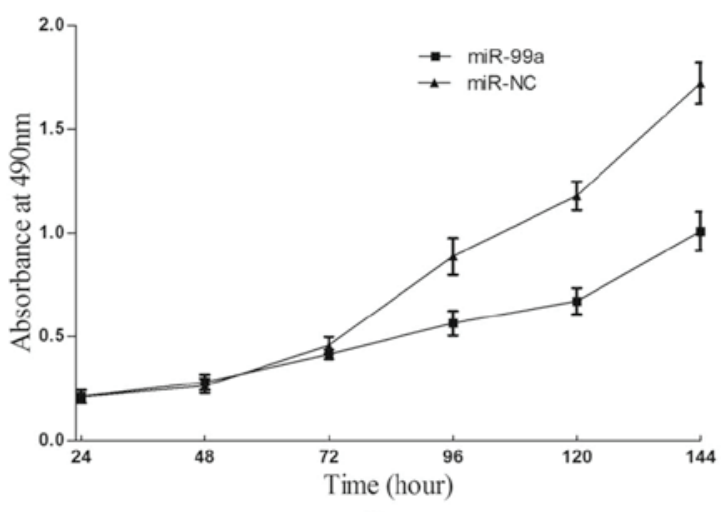

PC-3
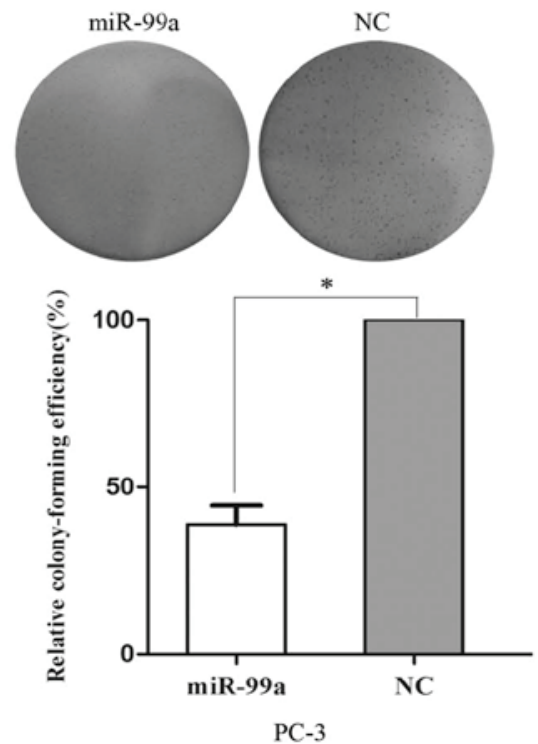

PC-3

Figure 2. Upregulation of miR-99a in prostate cancer DU145 and PC-3 cell lines suppresses cell proliferation and inhibits colony formation. (A) MTT assay determined cell proliferation in DU145 and PC-3 cells following upregulation of miR-99a. Increased miR-99a expression significantly suppressed cell proliferation in prostate cancer DU145 and PC-3 cell lines. " $\mathrm{P}<0.05$ compared with the NC. (B) Colony formation assay determined the effect of upregulated miR-99a on colony forming ability in prostate cancer DU145 and PC-3 cell lines. 2,000 cells were transfected with miR-99a or NC fixed with formalin and stained with methyl violet to show colony formation. The results indicated that up-regulation of miR-99a significantly suppressed colony formation ability in DU145 and PC-3 cells. "P<0.05 compared with NC. NC, negative control; miR-99a, microRNA 99a.
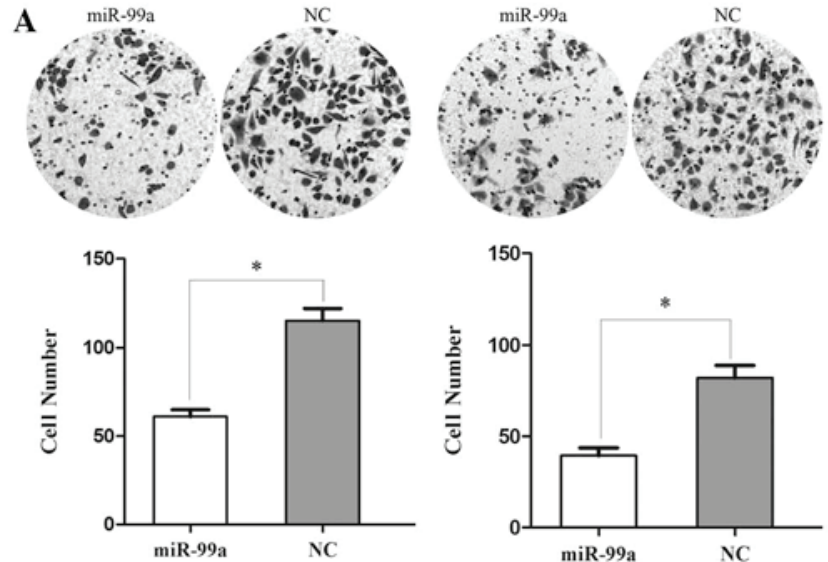

DU145 migration

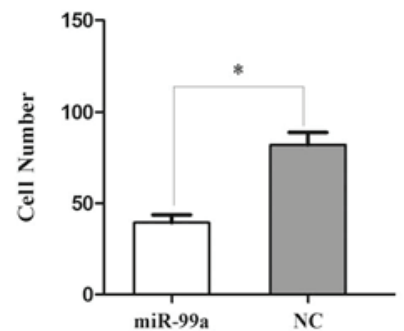

PC-3 migration
B
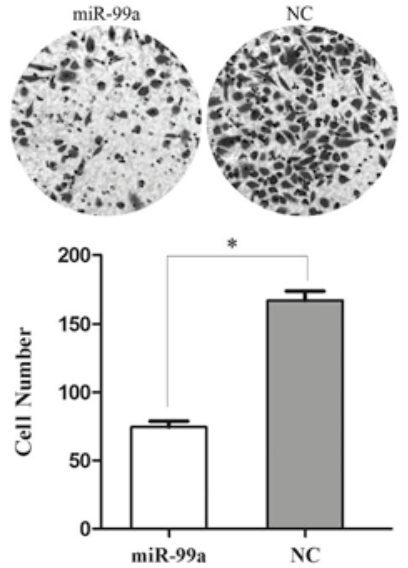

DU145 invasion
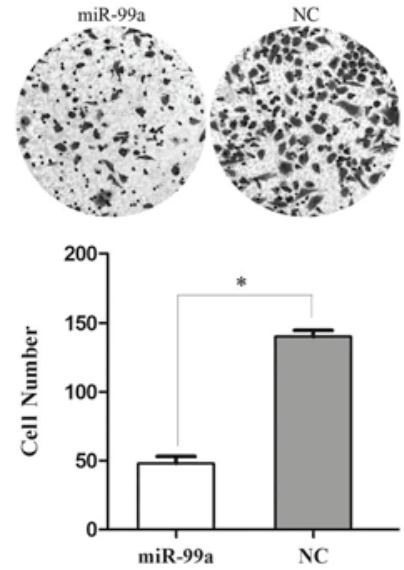

PC-3 invasion

Figure 3. Upregulation of miR-99a in prostate cancer DU145 and PC-3 cell lines inhibits cell migration and invasion. (A) Transwell chambers were used to detect cell migration in DU145 and PC-3 cell lines following the transfection of miR-99a or NC. Cells were incubated for $12 \mathrm{~h}$ and the number of miR-99a-transfected cells that passed through the Transwell membrane was significantly decreased. $\mathrm{P}<0.05$ compared with NC. (B) Transwell chambers coated with Matrigel ${ }^{\circledR}$ were used to detect cell invasion in DU145 and PC-3 cell lines following transfection of miR-99a or NC. Cells were incubated for 24 h, and the ability of miR-99a-transfected cells to pass through the Transwell membrane was significantly inhibited, therfore demonstrating decreased cell invasion. ${ }^{*} \mathrm{P}<0.05$ compare with NC. NC, negative control; miR-99a, microRNA 99a. 


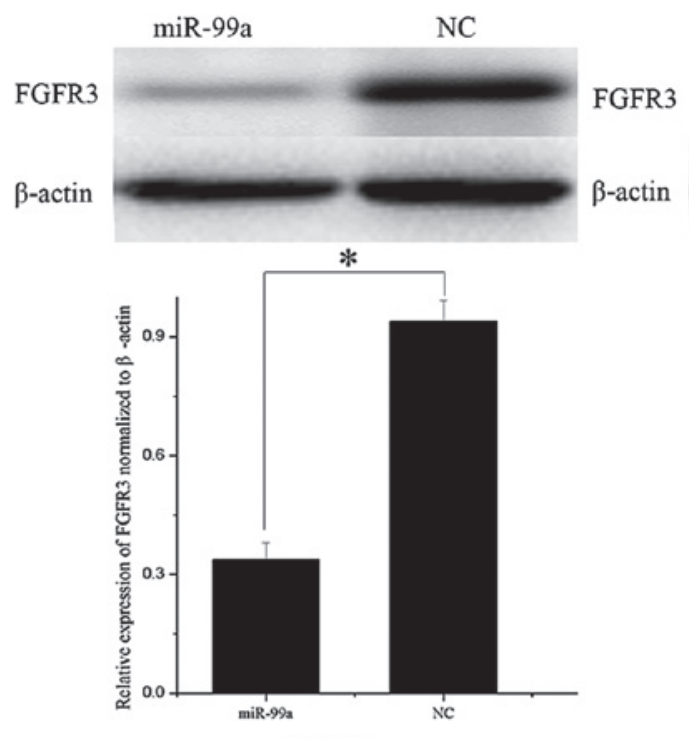

DU145

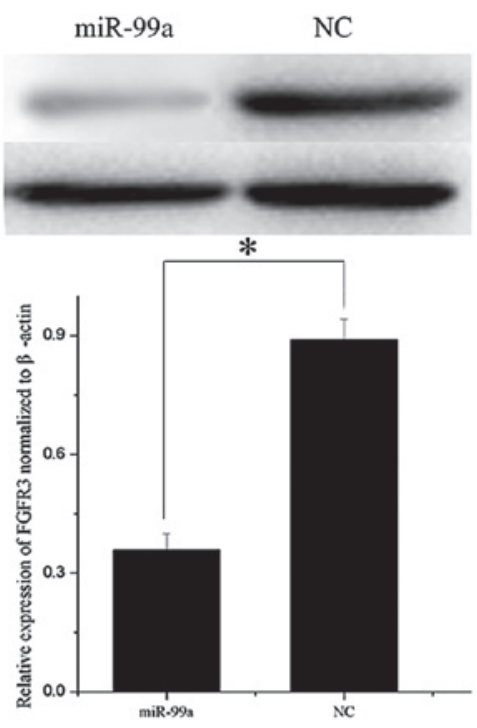

PC-3

Figure 4. Western blot analysis revealed decreased FGFR3 expression following transfection of miR-99a mimics in prostate cancer DU145 and PC-3 cells. Western blot analysis was performed $72 \mathrm{~h}$ following transfection of miR-99a into DU145 and PC-3 cells. Relative expression of FGFR3 was normalized to $\beta$-actin. FGFR3 expression was significantly reduced in prostate cancer DU145 and PC-3 cells following transfection of miR-99a. " $\mathrm{P}<0.05$ compared with NC. NC, negative control; miR-99a, microRNA 99a; FGFR3, fibroblast growth factor receptor 3.

A

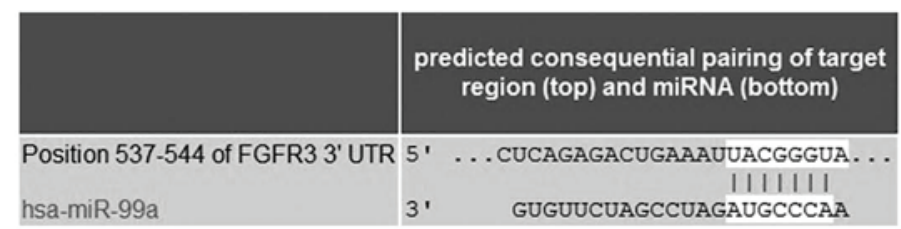

B

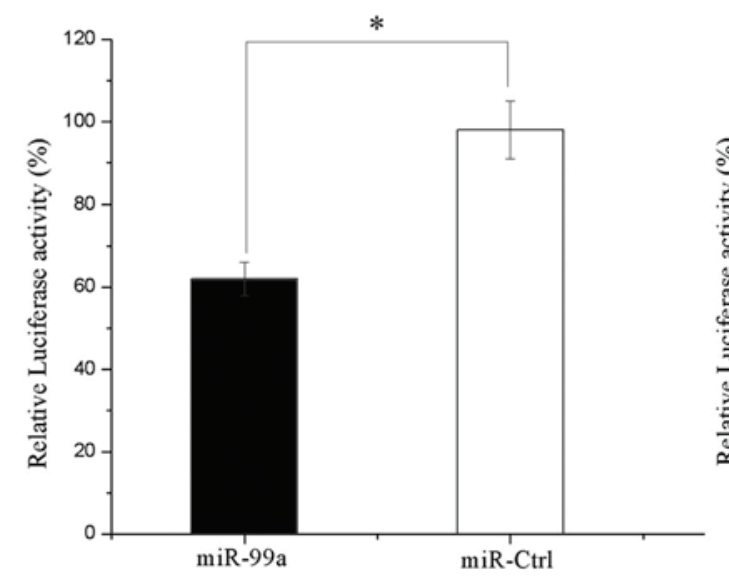

DU145

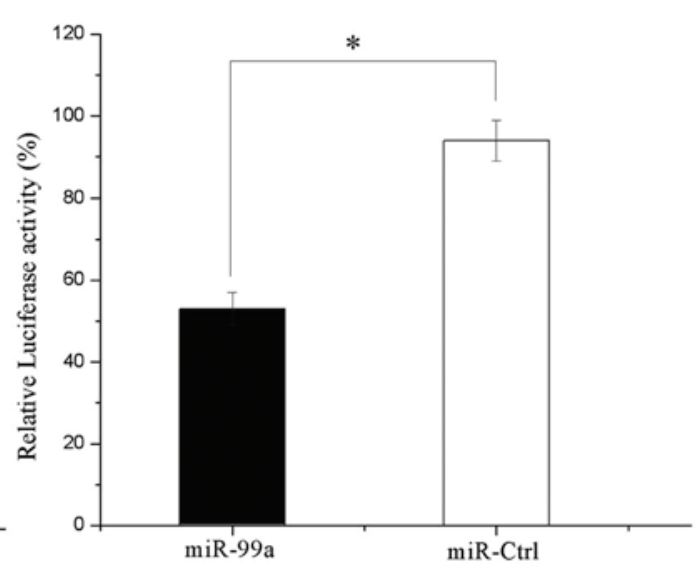

PC-3

Figure 5. miR-99a may directly target FGFR3 in vitro. (A) FGFR3 mRNA was assessed using TARGETSCAN, which revealed a miR-99a seven-nucleotide seed match at position 537-544 of the FGFR3 3'-UTR. (B) Luciferease reporter assays were performed to investigate whether FGFR3 is a target of miR-99a. Luciferase activity was significantly lowered when co-transfected with miR-99a and reporter plasmid in prostate cancer DU145 and PC-3 cell lines. FGFR3 may be a direct target of miR-99a in vitro. ${ }^{*} \mathrm{P}<0.05$ compared with NC. NC, negative control; miR-99a, microRNA 99a; mRNA, messenger RNA; FGFR3, fibroblast growth factor receptor 3; 3'-UTR, 3' untranslated region; hsa, Homo sapiens.

FGFR3 is downregulated following overexpression of miR-99a in DU145 and PC-3 cells. Western blot analysis was used to determine whether FGFR3 expression was altered following miR-99a transfection into prostate cancer DU145 and PC-3 cells. As shown in Fig. 4, the relative expression of FGFR3 was significantly downregulated in DU145 and PC-3 cell lines following overexpression of miR-99a $(\mathrm{P}<0.05)$. These results indicated that miR-99a reduced protein levels of FGFR3 in prostate cancer cells.

FGFR3 is a direct target gene of miR-99a in prostate cancer. In order to determine whether miR-99a targets the FGFR3 
3'-UTR, TARGETSCAN 5.2 and PICTAR were used to assess the complementarity of miR-99a to the FGFR3 3'-UTR. The results revealed that FGFR3 mRNA contained a miR-99a seven-nucleotide seed match at position 537-544 of the FGFR3 3'-UTR (Fig. 5A).

Luciferase reporter assays were performed to investigate whether FGFR3 is a target of miR-99a. As shown in Fig. 5B, overexpression of miR-99a suppressed FGFR3 3'UTR-luciferase activity by $36 \%$ in DU145 cells and by $43 \%$ in PC-3 cells $(\mathrm{P}<0.05)$. This therefore implied that FGFR3 may be a direct target of miR-99a in vitro.

\section{Discussion}

miRNAs are major regulatory molecules that govern numerous essential cellular functions, including proliferation, differentiation, cell cycle control and apoptosis (19).

Dysregulation of miRNAs has been reported to result in dysregulated gene expression of oncogenes and tumor suppressor genes, subsequently leading to human diseases such as cancer (15). The miR-99 family of miRNAs is comprised of three members, miR-99a, miR-99b and miR-100; the mature sequences of these three members are located on different chromosomes (20). miR-99a is transcribed from 21q21, a region that has been reported to be commonly deleted in human lung cancers (21). The upregulation of miR-99a has also been demonstrated in childhood acute myeloid leukemia (22). However, the role of miR-99a in various malignant diseases remains to be elucidated. Previous studies have reported that the downregulation of miR-99a contributed to tumorigenesis of several types of cancer, including prostate, bladder, hepatocellular and ovarian carcinoma, squamous cell carcinoma of the tongue, childhood adrenocortical tumors as well as lung cancer (17). The controversial effects of miR-99a can in part be explained by miRNAs binding to numerous 3'UTRs with complementary sites and therefore having multiple downstream target proteins. The role of microRNAs in cancer may be due to target proteins that have a tumor-promoting or tumor-inhibiting function.

miR-99a expression was reported to correlate with the survival rates of hepatocellular carcinoma patients; the upregulation of miR-99a was found to suppress growth of hepatocellular carcinoma cells via targeting the downstream proteins insulin-like growth factor 1 and mTOR (23). In renal cell carcinoma, it was demonstrated that downregulation of miR-99a resulted in increased tumorigenicity through targeting of the mTOR pathway; conversely, following the upregulation of miR-99a, tumorigenicity of renal cell carcinoma cell lines was suppressed in vitro and in vivo (17). In prostate cancer, miR-99a suppressed the expression of prostate-specific antigen and may be used as an indicator of the progression of prostate cancer (18). This therefore implied that the upregulation of miRNA-99a or the production of a synthetic analogue may provide effective therapeutic targets for the treatment of tumors that result from specific oncogene activation or overexpression.

The results of the present study indicated that miR-99a may function as a tumor suppressor via the direct targeting of FGFR3 in prostate cancer. Transfection of miR-99a into prostate cancer cell lines resulted in decreased cell viability and colony formation ability as well as reduced cell migration and invasion. This therefore suggested that miR-99a may have a potential therapeutic role through regulation of the oncogenic FGFR3 in prostate cancer patients.

The fibroblast growth factor (FGF) gene family consists of $>19$ genes encoding associated polypeptide mitogens. FGFs interact with a family of four distinct, high-affinity tyrosine kinase receptors, designated FGFR1-4 (24). FGFRs are composed of an extracellular domain that consists of three immunoglobulin-like domains and an intracellular tyrosine kinase domain. The affinity of FGF binding varies dependent on the FGFR (25); activation of FGFRs triggers different responses in different cell types, and these responses include proliferation, migration and differentiation as well as the inhibition of proliferation and/or cell death (26). Therefore, the role of deregulated FGF signaling in carcinogenesis has been the focus of numerous studies.

The family of FGFs and FGFRs are important in prostate organogenesis as well as the pathogenesis of prostate cancer (27). FGFR3 has been reported to have a major role in numerous types of cancer. The extracellular portion of FGFR3 interacts with FGF3, inducing trans autophosphorylation at the tyrosine residues of the cytoplasmic domain and subsequently stimulating intrinsic catalytic activity and activation of downstream signaling pathways (28). Several of these FGFR3-stimulated signal transduction pathways have been implicated in oncogenesis, including the Ras/extracellular-signal-regulated kinase/mitogen-activated protein kinase, the phospholipase $\mathrm{Cc} /$ protein kinase $\mathrm{C}$, phosphatidylinositol 3-kinase, and the signal transducers and activators of transcription pathways (29). FGFR signaling has also been reported to be involved in the activation of nuclear factor kappa-light-chain-enhancer of activated B cells $(30,31)$, the dysregulated activation of which is prevalent in human cancer $(32,33)$ and closely correlates with cancer hallmarks (34).

The overexpression of FGFR3 typically occurs through mutations within its extracellular and transmembrane domains as well as through overexpression of the wild type receptor (35). Hernández et al (36) reported that the highest recorded number of FGFR3 mutations was in patients with additional tumors as well as prostate cancer, i.e. bladder, skin, and colon tumors. In bladder cancers, mutations occurred predominantly in non-muscle invasive disease and significantly less frequently in muscle-invasive lesions. This therefore indicated that these mutations may be associated with a favorable course of the disease in non-invasive papillary bladder cancer (37). In prostate cancer, FGFR3 is expressed in the majority of benign prostatic hyperplasia (BPH) and prostate cancer. The expression pattern was reported to be predominantly epithelial with significant nuclear signals in BPH as well as the malignant prostate dysplasia (38). Hernández et al (36) reported an association between FGFR3 mutation frequency in low-grade prostate cancer and prostate cancer in patients with other coinciding malignancies. These results therefore indicated that FGFR3 may be a potential target for inhibition in prostate cancer.

The results of the present study revealed that miR-99a suppressed prostate cancer cell proliferation, colony formation ability, migration and invasion via the downregulation of 
FGFR3, therefore suggesting miR-99a and FGFR3 as targets of therapeutic drugs for prostate cancer.

In conclusion, to the best of our knowledge, the present study was the first to report that miR-99a reduced cell proliferation, colony formation ability, migration and invasion through regulation of FGFR3 in prostate cancer. The identification of candidate target genes of miR-99a may help to elucidate the carcinogenic mechanisms involved in prostate cancer. The results of the present study have promising therapeutic implications and therefore provide the basis for further studies for the treatment of prostate cancer. Future studies are required to address whether the potential of miR-99a may be fully realized in cancer treatment. If so, these may provide beneficial results for treatment of prostate cancer.

\section{References}

1. Siegel R, Naishadham D and Jemal A: Cancer statistics, 2013. CA Cancer J Clin 63: 11-30, 2013.

2. McGoldrick CA, Jiang YL, Paromov V, Brannon M, Krishnan K and Stone WL: Identification of oxidized protein hydrolase as a potential prodrug target in prostate cancer. BMC Cancer 14: 77, 2014.

3. Damber JE and Aus G: Prostate cancer. Lancet 371: 1710-1721, 2008.

4. Shavers VL, Underwood W and Moser RP: Race/ethnicity and the perception of the risk of developing prostate cancer. Am J Prev Med 37: 64-67, 2009.

5. Ma S, Chan YP, Kwan PS, et al: MicroRNA-616 induces androgen-independent growth of prostate cancer cells by suppressing expression of tissue factor pathway inhibitor TFPI-2. Cancer Res 71: 583-592, 2011.

6. Filipowicz W, Bhattacharyya SN and Sonenberg N: Mechanisms of post-transcriptional regulation by microRNAs: are the answers in sight? Nat Rev Genet 9: 102-114, 2008.

7. Bartel DP: MicroRNAs: target recognition and regulatory functions. Cell 136: 215-233, 2009.

8. Ambros V: The functions of animal microRNAs. Nature 431: 350-355, 2004

9. Calin GA and Croce CM: MicroRNA signatures in human cancers. Nat Rev Cancer 6: 857-866, 2006.

10. Esquela-Kerscher A and Slack FJ: Oncomirs - microRNAs with a role in cancer. Nature Rev Cancer 6: 259-269, 2006.

11. Lu J, Getz G, Miska EA, et al: MicroRNA expression profiles classify human cancers. Nature 435: 834-838, 2005.

12. Schaefer A, Stephan C, Busch J, Yousef GM and Jung K: Diagnostic, prognostic and therapeutic implications of microRNAs in urologic tumors. Nat Rev Urol 7: 286-297, 2010.

13. Fendler A, Jung M, Stephan C, et al: miRNAs can predict prostate cancer biochemical relapse and are involved in tumor progression. Int J Oncol 39: 1183-1192, 2011.

14. Schaefer A, Jung M,Kristiansen G, et al: MicroRNAs and cancer: current state and future perspectives in urologic oncology. Urol Oncol 28: 4-13, 2010

15. Zhou Y, Wu D, Tao J, Qu P, Zhou Z and Hou J: MicroRNA-133 inhibits cell proliferation, migration and invasion by targeting epidermal growth factor receptor and its downstream effector proteins in bladder cancer. Scand J Urol 47: 423-432, 2013.

16. Ventura A and Jacks T: MicroRNAs and cancer: short RNAs go a long way. Cell 136: 586-591, 2009.

17. Cui L, Zhou H, Zhao H, et al: MicroRNA-99a induces G1-phase cell cycle arrest and suppresses tumorigenicity in renal cell carcinoma. BMC Cancer 12: 546, 2012.
18. Sun D, Lee YS, Malhotra A, et al: miR-99 family of MicroRNAs suppresses the expression of prostate-specific antigen and prostate cancer cell proliferation. Cancer Res 71: 1313-1324, 2011.

19. Lerman G, Avivi C, Mardoukh C, et al: MiRNA expression in psoriatic skin: reciprocal regulation of hsa-miR-99a and IGF-1R. PloS One 6: e20916, 2011.

20. Sun J, Chen Z, Tan X, et al: MicroRNA-99a/100 promotes apoptosis by targeting mTOR in human esophageal squamous cell carcinoma. Med Oncol 30: 411, 2013.

21. Nagayama K, Kohno T, Sato M, Arai Y, Minna JD and Yokota J: Homozygous deletion scanning of the lung cancer genome at a 100-kb resolution. Genes Chromosomes Cancer 46: 1000-1010, 2007.

22. Li XJ, Luo XQ, Han BW, Duan FT, Wei PP and Chen YQ: MicroRNA-100/99a, deregulated in acute lymphoblastic leukaemia, suppress proliferation and promote apoptosis by regulating the FKBP51 and IGF1R/mTOR signalling pathways. Br J Cancer 109: 2189-2198, 2013.

23. Li D, Liu X, Lin L, et al: MicroRNA-99a inhibits hepatocellular carcinoma growth and correlates with prognosis of patients with hepatocellular carcinoma. J Biol Chem 286: 36677-36685, 2011.

24. Johnson DE and Williams LT: Structural and functional diversity in the FGF receptor multigene family. Adv Cancer Res 60: 1-41, 1993.

25. Kwabi-Addo B, Ropiquet F, Giri D and Ittmann M: Alternative splicing of fibroblast growth factor receptors in human prostate cancer. Prostate 46: 163-172, 2001.

26. Lafitte M, Moranvillier I, Garcia S, et al: FGFR3 has tumor suppressor properties in cells with epithelial phenotype. Mol Cancer 12: 83, 2013.

27. Dorkin TJ, Robinson MC, Marsh C, Neal DE and Leung HY: aFGF immunoreactivity in prostate cancer and its co-localization with bFGF and FGF8. J Pathol 189: 564-569, 1999.

28. Azab AK, Azab F, Quang P, et al: FGFR3 is overexpressed waldenstrom macroglobulinemia and its inhibition by Dovitinib induces apoptosis and overcomes stroma-induced proliferation. Clin Cancer Res 17: 4389-4399, 2011.

29. Wesche J, Haglund K and Haugsten EM: Fibroblast growth factors and their receptors in cancer. BiochemJ 437: 199-213, 2011.

30. Ettelaie C, Fountain D, Collier ME, Elkeeb AM, Xiao YP and Maraveyas A: Low molecular weight heparin downregulates tissue factor expression and activity by modulating growth factor receptor-mediated induction of nuclear factor- $\kappa \mathrm{B}$. Biochim Biophys Acta 1812: 1591-1600, 2011.

31. Lungu G, Covaleda L, Mendes O, Martini-Stoica H and Stoica G: FGF-1-induced matrix metalloproteinase-9 expression in breast cancer cells is mediated by increased activities of NF-kappaB and activating protein-1. Mol Carcinog 47: 424-435,2008.

32. Chaturvedi MM, Sung B, Yadav VR, Kannappan R, and Aggarwal BB: NF- $\kappa$ B addiction and its role in cancer: 'one size does not fit all'. Oncogene 30: 1615-1630, 2011.

33. Perkins ND: The diverse and complex roles of NF- $\mathrm{KB}$ subunits in cancer. Nat Rev Cancer 12: 121-132, 2012.

34. Hanahan D and Weinberg RA: The hallmarks of cancer. Cell 100: $57-70,2000$.

35. Tomlinson DC, Hurst CD and Knowles MA: Knockdown by shRNA identifies S249C mutant FGFR3 as a potential therapeutic target in bladder cancer. Oncogene 26: 5889-5899, 2007.

36. Hernández S, de Muga S, Agell L, et al: FGFR3 mutations in prostate cancer: association with low-grade tumors. Mod Pathol 22: 848-856, 2009.

37. Hernández S, López-Knowles E, Lloreta J, et al: Prospective study of FGFR3 mutations as a prognostic factor in nonmuscle invasive urothelial bladder carcinomas. J Clin Oncol 24: 3664-3671, 2006.

38. Gowardhan B, Douglas DA, Mathers ME, McKie AB, McCracken SR, Robson CN and Leung HY: Evaluation of the fibroblast growth factor system as a potential target for therapy in human prostate cancer. Br J Cancer 92: 320-327, 2005. 